\title{
Disposição de resíduo de ETA em sistema de tratamento aeróbio de esgoto seguido de lagoa de sedimentação
}

\author{
Disposal of water treatment residue on aerobic proccess of a \\ wastewater treatment plant
}

Paulo Sergio Scalize* | Luiz Di Bernardo | Lorena Acelina Soares | Luis Rodrigo Fernandes Baumann

Data de entrada: 11/12/2013 | Data de aprovação: 23/05/2014

DOI: http://dx.doi.org/10.4322/dae.2014.130

Resumo

O trabalho foi realizado utilizando uma instalação piloto (ETEP) constituída de dois módulos de tratamento aeróbio, cada um composto por lagoa de aeração seguida de lagoa de sedimentação, ambos com parâmetros hidráulicos similares ao da ETE, sendo que um funcionou recebendo somente esgoto afluente à ETE. No outro módulo, além de esgoto, foi adicionado resíduo de uma estação de tratamento de água (RETA) que utilizava cloreto férrico como coagulante primário. Por meio de teste estatístico, com nível de significância de 10\%, foi verificado que a aplicação de RETA melhorou a qualidade do efluente final do módulo da ETEP em relação aos parâmetros DQO, SST, turbidez, cor aparente, nitrito, NTK e fósforo total. Os demais parâmetros também resultaram melhores, porém com níveis de significância superiores, com exceção do cloreto, nitrato e condutividade, que praticamente ficaram inalterados.

Palavras-chave: Resíduos de ETA, lodo, resíduo, estação de tratamento de água.

\begin{abstract}
This work was carried out in an aerobic pilot plant consisted of modules, each one with an aerated lagoon in series with a sedimentation lagoon, both with hydraulic characteristics similar to the city wastewater treatment plant. Module 1 received water treatment residue besides sewage while module 1 received only sewage. Through the use of statistics methods with significance level of $10 \%$, it was observed that the module 1, which received the water treatment residue produced a better effluent quality in terms of COD, TSS, turbidity, apparent color, nitrite, TNK and total phosphorus. Other parameters investigated in the effluent of module 1 also resulted better, but with a higher level of significance, except for chlorite, nitrate and conductivity, which practically remained unchanged.
\end{abstract}

Key-words: Water treatment residue, sludge, residue, water treatment plant

*Curriculum dos autores - ver página 86 


\section{Introdução}

Apesar de existirem diversos métodos para tratamento e disposição dos resíduos gerados em estação de tratamento de água - RETA, é extremamente importante encontrar novas alternativas, visando à proteção do meio ambiente com menores custos envolvidos nos processos e operações utilizados.

Segundo Januário et al. (2007), para os RETAs da Região Metropolitana de São Paulo, os métodos disponíveis de uso e disposição final poderiam ser: i) aterros; ii) aplicação no solo para agricultura e para recuperação de áreas degradadas; iii) produção de artefatos de cerâmica, de cimento ou concreto; iv) lançamento em ETE; v) recuperação de coagulante, vi) secagem e incineração. Existem vários trabalhos na literatura nacional e internacional sobre o tema, tais como disposição em ETEs (Carvalho, 2000; Scalize, 2003; Ferreira Filho e Waelkens, 2009; Scalize e Di Bernardo, 2011; Di Bernardo et al., 2011), fabricação de materiais cerâmicos (Oliveira e Holanda, 2008; Tartari et al., 2011) e de tijolos (Teixeira et al., 2006), recuperação de áreas degradadas (Teixeira et al., 2005 e 2007; Moreira et al., 2009), aplicações agrícolas (Dayton and Basta, 2001; Botero et at, 2009; Park et al., 2009; $\mathrm{OH}$ et al., 2010; Figueiredo Neto et al., 2012) e minimização de RETA (Achon et al., 2008; Ferreira Filho e Waelkens, 2009).

O lançamento de RETA em ETEs, via rede coletora de esgoto ou tubulação exclusiva, é um procedimento que surge como proposta atraente, visto que elimina a implantação de sistemas de tratamento de RETA junto à ETA. Entretanto, algumas interferências podem ocorrer nas unidades da ETE, de maneira que tal procedimento deve ser criteriosamente analisado. Para evitar possíveis interferências no desempenho das ETEs e disposição de seu lodo, devem ser avaliados alguns fatores, tais como: sobrecargas hidráulicas, orgânicas, de sólidos; toxicidade aos organismos responsáveis pela depuração; separação sólido-líquido; processos biológicos de tratamento; adensamento, desaguamento e disposição final do lodo de ETEs. Carvalho (2000), concluiu em sua pesquisa que o lançamento do resíduo de ETA que utilizava cloreto férrico como coagulante, em ETE com decantação primária, em dosagens de até 225 mg ST RETA/L esgoto, não causava efeitos prejudiciais, apontando que essa alternativa poderia aumentar a eficiência de remoção de DQO, fósforo total e coliformes totais e E. coli. Scalize (2003), realizando estudos em colunas de sedimentação, simulando o lançamento de RETA (proveniente de uma ETA que utilizava sulfato de alumínio como coagulante primário) em decantadores primários de uma ETE, concluiu que o efluente final não era prejudicado, podendo ocorrer melhoras, no entanto, os ensaios para verificar a toxicidade durante digestão anaeróbica do lodo formado nos decantadores primários indicaram uma redução de $18 \%$ na produção de metano, influenciando negativamente sobre os microrganismos metanogênicos. Scalize e Di Bernardo (2011), ao realizarem ensaios em coluna de sedimentação, relataram em seu trabalho que não ocorreria deterioração no efluente dos decantadores primários de uma ETE, uma vez que a qualidade do sobrenadante nas colunas de sedimentação não foi prejudicada com a presença de RETA. Ferreira e Waelkens (2009) relatam em seu trabalho que uma eventual disposição de RETA da ETA Boa Vista na ETE Barueri deverá elevar o teor de Fe e Al no lodo desidratado. Di Bernardo et al. (2011) mencionam que as ETEs das cidades de Franca e Araraquara recebem RETA e não apresentam quaisquer problemas operacionais, o que indica a viabilidade de tal procedimento.

Carvalho (2000); Scalize (2003) e Scalize e Di Bernardo (2011), recomendam também que, após a equalização dos RETAs, sejam lançados em uma rede coletora de esgotos ou encaminhados por meio de tubulação exclusiva à ETE. Di Bernardo et al. (2011) relatam que é imprescindível o levantamento das vazões mínimas, médias e máximas de RETA e de esgoto a ser tratado pelas ETEs, a fim de minimizar os impactos que poderiam surgir, pois as cidades tem sistemas operacionais diferenciados podendo ser atendidas por uma ou mais ETA e ETE.

A presente pesquisa teve como objetivo verificar os efeitos provocados pelo lançamento de RETA em uma ETE em escala piloto, constituída de lagoa aerada seguida por lagoa de sedimentação, com características hidráulicas similares à da ETE da cidade.

\section{Metodologia}

O RETA utilizado na pesquisa foi obtido em uma ETA de ciclo completo que utilizava cal para ajuste do $\mathrm{pH}$ de coagulação e cloreto férrico como coagulante. O RETA utilizado apresentou 
teor de SST de 1,0 g/L, composto por 54,3\% de água de descarga de decantador (SST $=1606$ $\mathrm{mg} / \mathrm{L}$ ) e $45,7 \%$ de água de lavagem de filtro (SST $=298 \mathrm{mg} / \mathrm{L}$ ), de acordo com os dados operacionais da ETA. O esgoto sanitário utilizado na pesquisa, também da mesma cidade, apresentou valores médios de DQO, DBO, SST, fosfato total, Nitrogênio amoniacal e Nitrogênio Total Kjeldahl iguais a 740; 320; 216; 8,3; 14,2 e 25,4 mg/L, respectivamente.

Na pesquisa foi utilizada uma instalação piloto, ilustrada na Figura 1, composta por dois módulos de lagoas, cada um com uma lagoa aerada LA, com volume útil de 30 L e tempo de detenção de 3 dias, em série com uma lagoa de sedimentação - LS, com volume útil de $10 \mathrm{~L}$ e tempo de detenção de 1,0 dia. A alimentação (constante) das lagoas de aeração, com esgoto sanitário bruto, foi realizada ininterruptamente por meio de bombas dosadoras, com uma vazão de 6,94 $\mathrm{mL} / \mathrm{min}$. Durante a operação do sistema, foram realizadas leituras de oxigênio dissolvido (OD) no início, meio e final das lagoas de aeração, resultando em qualquer ponto, entre 1,5 e $1,7 \mathrm{mg} / \mathrm{L}$, decorrente da introdução de ar proveniente de um compressor com vazão regulada por meio de rotâmetros nas duas linhas que alimentavam cada módulo de lagoas da ETEP.

Antes de iniciar a adição do RETA, os dois módulos de lagoas da ETEP funcionaram 30 dias recebendo apenas esgoto sanitário. Após esse período, foi iniciada a adição de RETA na LA1, sendo realizada em alíquotas de 82,5 mL, 6 vezes ao dia, em um período de operação de 90 dias, totalizando $44,55 \mathrm{~L}$.

Foram realizadas caracterizações dos afluentes aos dois módulos de lagoas da ETEP e dos efluentes na saída de cada LS em intervalos de 7 dias, totalizando 13 amostras. Os parâmetros físico-químicos pesquisados foram DBO, DQO, $\mathrm{pH}$, condutividade elétrica, sólidos totais (ST), sólidos totais fixos (STF), sólidos totais voláteis (STV), sólidos suspensos totais (SST), sólidos suspensos fixos (SSF), sólidos suspensos voláteis (SSV), sólidos dissolvidos totais (SDT), sólidos dissolvidos fixos (SDF), sólidos dissolvidos voláteis (SDV), turbidez, cor aparente, cloreto, fósforo total, amônio, nitrito, nitrato e Nitrogênio Total Kjeldahl (NTK), cujas medidas foram realizadas conforme Standard Methods (APHA-WEF-AWWA, 2005).

Os resultados obtidos nas caracterizações dos efluentes das lagoas de sedimentação foram submetidos a um tratamento estatístico por meio da aplicação do Teste de Normalidade Shapiro Wilk e teste de variância com nível de significância de 5\%. Para os resultados que apresentaram evidências de normalidade, foi aplicado o Teste t com $10 \%$ de significância e quando a normalidade dos dados foi rejeitada, foi aplicado o Teste de Wilcoxon com $10 \%$ de significância. O Teste de Normalidade Shapiro Wilk, Teste de Variância, Teste de Wilcoxon e Teste t constam de Bussab \& Morettin (2002).

A microfauna foi avaliada no licor misto das LA por meio de exames microscópicos qualitativos,
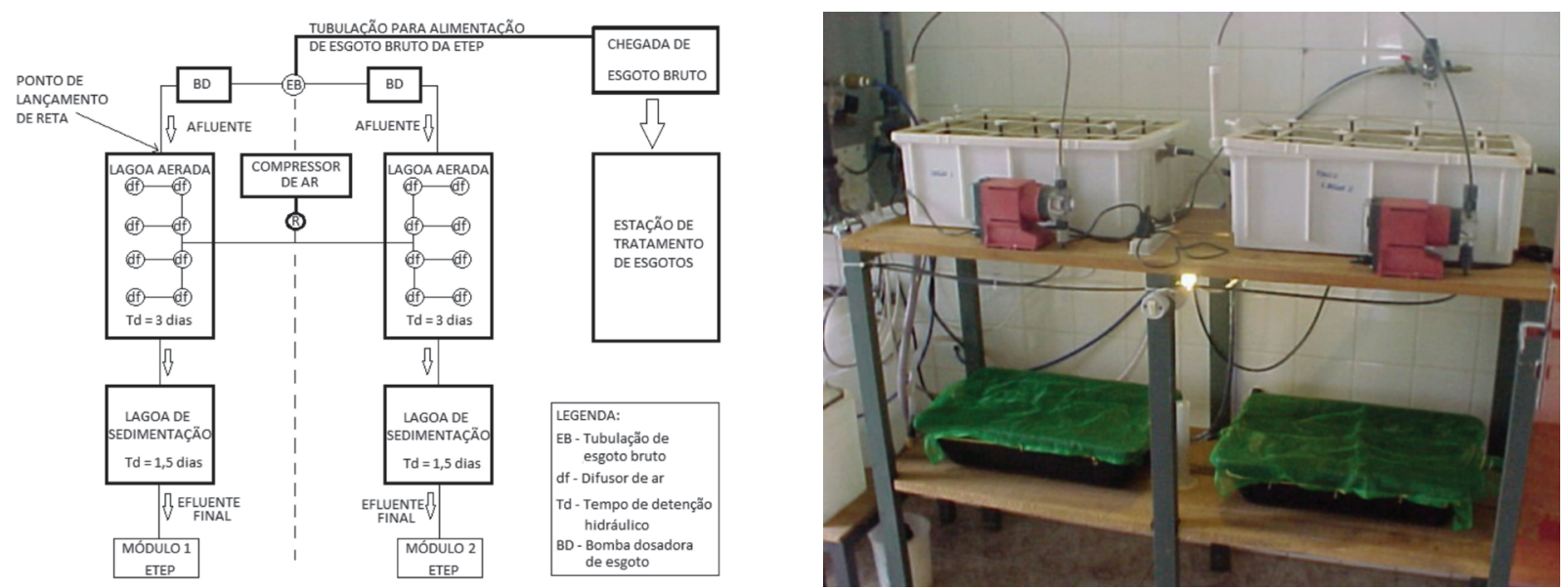

Figura 1 - Esquema e foto da instalação piloto (ETEP) utilizada na pesquisa. 
antes do início da introdução de RETA e após os 90 dias de operação, visando avaliar as possíveis diferenças entre os grupos predominantes de organismos presentes.

Em duas ocasiões (78 e 90 dias de operação) foi determinada a taxa de consumo de oxigênio (TCO) no licor misto das lagoas de aeração dos dois módulos da ETEP, conforme metodologia proposta por Von Sperling (1997). Nesse ensaio, os valores de OD foram medidos com auxílio de um oxímetro da marca Digimed, em intervalos de 1 minuto.

Ao final dos 120 dias $(30$ + 90) de operação, a alimentação foi interrompida e os lodos formados nas lagoas LS1 e LS2 foram removidos e submetidos à caracterização físico-química, por meio da medição de DQO, pH, série de sólidos e metais (ferro, níquel, zinco, chumbo, cádmio, cobre, cromo e cobalto), conforme o Standard Methods (APHA-WEF-AWWA, 2005). Posteriormente, foram realizados ensaios para determinação da Resistência Específica - RE, conforme Standard Methods (APHA-WEF-AWWA, 2005) e ensaios de centrifugação segundo Reali et al. (1999), sendo as tortas resultantes nesses ensaios submetidas a ensaios de lixiviação e solubilização de acordo com a NBR 10004 (ABNT, 2004).

Os sobrenadantes dos ensaios de centrifugação foram caracterizados em termos de DQO, turbidez e cor aparente, e medidos os metais ferro, níquel, zinco, chumbo, cádmio, cobre, cromo e cobalto no sedimento.

\section{Resultado e discussão}

Visando avaliar as interferências ou vantagens decorrentes da introdução de RETA em um dos módulos de LA-LS, foram realizadas caracterizações do afluente a cada módulo de lagoas e do efluente das LS durante os 90 dias de operação da ETEP.

Os resultados obtidos estão contidos na Tabela 1, e os valores mínimos, máximos, médios, bem como desvio padrão e coeficiente de variação estão inseridos na Tabela 2 , apresentados do $1^{\circ}$ ao $90^{\circ}$ dia e em separado do $1^{\circ}$ ao $42^{\circ}$ e do $43^{\circ}$ ao $90^{\circ}$, pois foram observadas maiores variações nos resultados na etapa inicial. No tratamento estatístico (Tabela 3) dos resultados obtidos durante os 90 dias de operação das ETEP, a hipótese de normalidade foi rejeitada em 71,4\% dos parâmetros pesquisados com nível de significância de $5 \%$, sendo nesse caso aplicado o teste de Sha- piro Wilk, e nos demais, o Teste t. Os resultados evidenciaram que os valores de DQO, SST, SSF, SSV, turbidez, cor aparente, nitrito, NTK e fósforo total, apresentaram menores concentrações nos efluentes provenientes da LS1 (que recebeu RETA). Os demais parâmetros não apresentaram indícios de influência com aplicação do RETA, e, caso fosse utilizado nível de significância maior, outros parâmetros teriam melhoras favoráveis, com exceção de condutividade, ST, cloreto e nitrato que praticamente não apresentaram diferenças.

Considerando que do $1^{\circ}$ ao $42^{\circ}$ dia pode ter ocorrido aclimatação nas lagoas, os resultados foram tratados estatisticamente divididos em dois blocos, sendo o primeiro até o $42^{\circ}$ dia e o segundo do $43^{\circ}$ ao $90^{\circ}$ dia. No primeiro bloco (Tabela 4) pode ser observada influência negativa quanto a SV e SDV, ou seja, o efluente da LS1 apresentou maiores valores desses parâmetros, não tendo sido observadas interferências nos demais parâmetros, com exceção de cor aparente e fósforo total que apresentaram valores menores. No segundo bloco (Tabela 5), foi notada evidência de normalidade em quase todos os parâmetros e melhor eficiência do módulo LA1-LS1 com relação aos parâmetros SST, SSF, SSV, turbidez, cor aparente, nitrito, NTK e fósforo total.

Os resultados obtidos evidenciaram que as variáveis pesquisadas no efluente da LS1, proveniente do módulo LA1/LS1 (que recebeu RETA), não sofreram interferências negativas, com produção de efluente de melhor qualidade de praticamente todos os parâmetros pesquisados. Somente para cloreto, condutividade e nitrato não ocorreu redução devido a aplicação do RETA, mas não houve piora do efluente.

A melhora no sobrenadante de decantadores primários de uma ETE que recebeu RETA foi evidenciada por Carvalho (2000), para os parâmetros DQO e fósforo total, e por Scalize (2003), para os parâmetros cor, turbidez, SST, SSV e DQO. O fósforo total foi reduzido no módulo LA1-LS1 (que recebeu RETA) conforme pode ser visto na Figura 2. A remoção de fósforo com utilização de RETA também foi constatada por Chao et al. (2011) em sua pesquisa. A concentração de fósforo total nas amostras provenientes da LS2 foi de $10,17 \%$ a $31,87 \%$ superior ao do efluente da LS1, evidenciando que o RETA auxiliou na melhora da qualidade do efluente. 


\begin{tabular}{|c|c|c|c|c|c|c|c|c|c|c|c|c|c|}
\hline \multirow{2}{*}{ Parâmetro } & \multicolumn{13}{|c|}{ Dia de operação } \\
\hline & 7 & 14 & 21 & 28 & 35 & 42 & 49 & 56 & 63 & 70 & 77 & 84 & 90 \\
\hline DQO-LS1 (mg/L) & 36,5 & 45,8 & 41,0 & 61,8 & 86,0 & 65,7 & 89,8 & 59,4 & 62,6 & 54,2 & 51,8 & 46,2 & 43,4 \\
\hline DQO-LS2 (mg/L) & 39,4 & 50,4 & 58,1 & 84,0 & 123,1 & 78,0 & 102,7 & 65,3 & 74,2 & 69,1 & 68,0 & 53,0 & 52,8 \\
\hline DBO-LS1 (mg/L) & 12,0 & 14,0 & 33,0 & 17,1 & 26,0 & 12,0 & 25,0 & 14,0 & 21,0 & 19,0 & 18,0 & 16,0 & 26,0 \\
\hline DBO-LS2 (mg/L) & 16,0 & 20,0 & 31,0 & 20,7 & 32,2 & 29,0 & 20,5 & 19,0 & 25,0 & 20,0 & 22,0 & 17,0 & 27,5 \\
\hline pH-LS1 & 6,6 & 6,8 & 6,6 & 6,9 & 7,2 & 7,2 & 7,5 & 7,3 & 7,2 & 7,1 & 7,2 & 7,3 & 7,4 \\
\hline pH-LS2 & 7,0 & 6,9 & 6,4 & 6,6 & 7,3 & 7,0 & 7,2 & 7,2 & 7,1 & 7,2 & 7,2 & 7,2 & 7,3 \\
\hline $\begin{array}{l}\text { Condutividade-LS1 } \\
(\mu \mathrm{s} / \mathrm{cm})\end{array}$ & 482 & 527 & 367 & 418 & 490 & 422 & 499 & 500 & 529 & 486 & 564 & 578 & 680 \\
\hline $\begin{array}{l}\text { Condutividade-LS2 } \\
(\mu \mathrm{s} / \mathrm{cm})\end{array}$ & 480 & 560 & 389 & 412 & 481 & 418 & 506 & 491 & 544 & 480 & 572 & 580 & 673 \\
\hline ST-LS1 (mg/L) & 517 & 560 & 591 & 410 & 716 & 928 & 334 & 450 & 311 & 431 & 374 & 355 & 321 \\
\hline ST-LS2 (mg/L) & 573 & 655 & 388 & 434 & 479 & 457 & 391 & 365 & 346 & 412 & 397 & 388 & 384 \\
\hline STF-LS1 (mg/L) & 209 & 325 & 282 & 142 & 313 & 325 & 264 & 215 & 198 & 255 & 260 & 245 & 221 \\
\hline STF-LS2 (mg/L) & 295 & 283 & 313 & 330 & 277 & 292 & 254 & 235 & 220 & 265 & 278 & 260 & 270 \\
\hline STV-LS1 (mg/L) & 308 & 235 & 309 & 268 & 403 & 603 & 70 & 235 & 113 & 176 & 114 & 110 & 100 \\
\hline STV-LS2 (mg/L) & 278 & 372 & 75 & 104 & 202 & 165 & 137 & 130 & 126 & 147 & 119 & 128 & 114 \\
\hline SDT-LS1 (mg/L) & 509 & 554 & 574 & 403 & 698 & 922 & 319 & 442 & 305 & 422 & 367 & 348 & 315 \\
\hline SDT-LS2 (mg/L) & 563 & 645 & 374 & 425 & 448 & 441 & 360 & 347 & 330 & 397 & 382 & 372 & 370 \\
\hline SDF-LS1 (mg/L) & 207 & 324 & 280 & 141 & 308 & 324 & 260 & 214 & 196 & 252 & 258 & 243 & 220 \\
\hline SDF-LS2 (mg/L) & 294 & 282 & 309 & 328 & 272 & 291 & 249 & 231 & 217 & 260 & 274 & 256 & 266 \\
\hline SDV-LS1 (mg/L) & 302 & 230 & 294 & 262 & 390 & 598 & 59 & 228 & 109 & 170 & 109 & 105 & 95 \\
\hline SDV-LS2 (mg/L) & 269 & 363 & 65 & 97 & 176 & 150 & 111 & 116 & 113 & 137 & 108 & 116 & 104 \\
\hline SST-LS1 (mg/L) & 8 & 6 & 17 & 7 & 18 & 6 & 15 & 8 & 6 & 9 & 7 & 7 & 6 \\
\hline SST-LS2 (mg/L) & 10 & 10 & 14 & 9 & 31 & 16 & 31 & 18 & 16 & 15 & 15 & 16 & 14 \\
\hline SSF-LS1 (mg/L) & 2 & 1 & 2 & 1 & 5 & 1 & 4 & 1 & 2 & 3 & 2 & 2 & 1 \\
\hline SSF-LS2 (mg/L) & 1 & 1 & 4 & 2 & 5 & 1 & 5 & 4 & 3 & 5 & 4 & 4 & 4 \\
\hline SSV-LS1 (mg/L) & 6 & 5 & 15 & 6 & 13 & 5 & 11 & 7 & 4 & 6 & 5 & 5 & 5 \\
\hline SSV-LS2 (mg/L) & 9 & 9 & 10 & 7 & 26 & 15 & 26 & 14 & 13 & 10 & 11 & 12 & 10 \\
\hline Turbidez-LS1 (UT) & 15 & 16 & 57 & 23 & 33 & 19 & 24 & 20 & 25 & 26 & 17 & 18 & 22 \\
\hline Turbidez-LS2 (UT) & 15 & 27 & 52 & 33 & 39 & 32 & 34 & 26 & 31 & 29 & 24 & 22 & 23 \\
\hline Cor Aparente-LS1 (uH) & 33 & 42 & 61 & 36 & 43 & 33 & 45 & 46 & 44 & 42 & 30 & 32 & 36 \\
\hline Cor Aparente-LS2 (uH) & 32 & 55 & 77 & 57 & 63 & 51 & 49 & 61 & 50 & 51 & 36 & 41 & 39 \\
\hline Cloreto-LS1 (mg/L) & 39,2 & 41,2 & 40,2 & 39,2 & 41,2 & 45,0 & 43,1 & 48,2 & 39,1 & 41,2 & 43,6 & 44,8 & 52,6 \\
\hline Cloreto-LS2 (mg/L) & 37,3 & 42,1 & 44,0 & 40,2 & 41,2 & 45,0 & 42,1 & 47,1 & 40,0 & 40,6 & 44,1 & 45,1 & 51,7 \\
\hline Amônio-LS1 (mg/L) & 0,4 & 0,2 & 0,4 & 0,1 & 0,0 & 0,0 & 0,0 & 0,1 & 0,1 & 0,2 & 0,1 & 0,1 & 0,0 \\
\hline Amônio-LS2 (mg/L) & 0,0 & 0,3 & 0,5 & 2,5 & 4,0 & 0,0 & 0,2 & 0,4 & 0,1 & 0,2 & 0,2 & 0,0 & 0,0 \\
\hline Nitrato-LS1 (mg/L) & 7,7 & 9,2 & 16,0 & 14,3 & 14,1 & 17,4 & 12,1 & 11,1 & 9,5 & 7,7 & 9,5 & 6,7 & 4,7 \\
\hline Nitrato-LS2 (mg/L) & 8,9 & 9,1 & 20,4 & 17,7 & 8,1 & 10,1 & 10,2 & 12,1 & 8,8 & 8,8 & 9,2 & 7,8 & 8,5 \\
\hline Nitrito-LS1 (mg/L) & 0,5 & 0,5 & 0,5 & 0,4 & 0,2 & 0,3 & 0,2 & 0,2 & 0,2 & 0,3 & 0,3 & 0,2 & 0,1 \\
\hline Nitrito-LS2 (mg/L) & 0,3 & 0,5 & 0,4 & 0,3 & 0,5 & 0,4 & 0,5 & 0,4 & 0,4 & 0,4 & 0,4 & 0,4 & 0,5 \\
\hline NTK-LS1 (mg/L) & NR & NR & 5,9 & NR & NR & 2,5 & 5,0 & 5,2 & 5,2 & 5,7 & 5,0 & 4,7 & 5,0 \\
\hline NTK-LS2 (mg/L) & NR & NR & 5,0 & NR & NR & 4,2 & 4,2 & 10,2 & 9,4 & 8,8 & 10,2 & 9,6 & 12,6 \\
\hline Fosforo total-LS1 (mg/L) & 5,3 & 5,5 & 4,7 & 3,8 & 4,5 & 4,5 & 5,0 & 4,5 & 4,9 & 5,0 & 5,2 & 4,9 & 5,3 \\
\hline Fosforo total-LS2 (mg/L) & 5,9 & 6,4 & 6,3 & 4,6 & 5,9 & 5,8 & 6,7 & 6,6 & 6,2 & 6,3 & 6,7 & 5,9 & 6,5 \\
\hline
\end{tabular}

Tabela 1 - Resultados dos parâmetros físicos e químicos dos efluentes provenientes das Lagoas LS1 e LS2 durante a operação da ETEP. 


\begin{tabular}{|c|c|c|c|c|c|c|c|c|c|c|c|c|c|c|c|}
\hline \multirow[b]{3}{*}{ Parâmetro } & \multicolumn{15}{|c|}{ Período de operação (dia) } \\
\hline & \multicolumn{5}{|c|}{$1^{\circ}$ ao $42^{\circ}$} & \multicolumn{5}{|c|}{$43^{\circ}$ ao $90^{\circ}$} & \multicolumn{5}{|c|}{$1^{\circ}$ ao $90^{\circ}$} \\
\hline & 龸 & 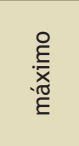 & हूँ & 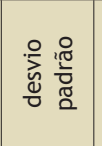 & 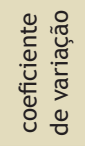 & 竘 & 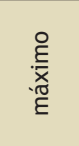 & : & 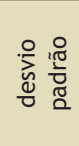 & 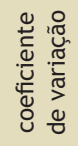 & 亳 & 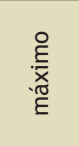 & 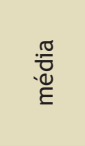 & 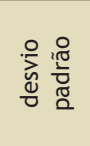 & 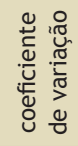 \\
\hline DQO-LS1 (mg/L) & 36,5 & 86,0 & 56,1 & 18,64 & 0,332 & 43,4 & 89,8 & 58,2 & 15,49 & 0,266 & 36,5 & 89,8 & 57,2 & 16,30 & 0,285 \\
\hline DQO-LS2 (mg/L) & 39,4 & 123,1 & 72,2 & 30,05 & 0,416 & 52,8 & 102,7 & 69,3 & 16,81 & 0,243 & 39,4 & 123,1 & 70,6 & 22,80 & 0,323 \\
\hline DBO-LS1 (mg/L) & 12,0 & 33,0 & 19,0 & 8,62 & 0,453 & 14,0 & 26,0 & 19,9 & 4,45 & 0,224 & 12,0 & 33,0 & 19,5 & 6,41 & 0,329 \\
\hline DBO-LS2 (mg/L) & 16,0 & 32,2 & 24,8 & 6,75 & 0,272 & 17,0 & 27,5 & 21,6 & 3,61 & 0,167 & 16,0 & 32,2 & 23,1 & 5,33 & 0,231 \\
\hline pH-LS1 & 6,6 & 7,2 & 6,9 & 0,27 & 0,039 & 7,1 & 7,5 & 7,3 & 0,13 & 0,018 & 6,6 & 7,5 & 7,1 & 0,29 & 0,041 \\
\hline pH-LS2 & 6,4 & 7,3 & 6,9 & 0,31 & 0,046 & 7,1 & 7,3 & 7,2 & 0,06 & 0,008 & 6,4 & 7,3 & 7,0 & 0,27 & 0,038 \\
\hline $\begin{array}{l}\text { Condutividade } \\
\text { LS1 }(\mu \mathrm{s} / \mathrm{cm})\end{array}$ & 367 & 527 & 451,0 & 58,73 & 0,130 & 486 & 680 & 548,0 & 67,69 & 0,124 & 367 & 680 & 503,2 & 79,13 & 0,157 \\
\hline $\begin{array}{l}\text { Condutividade } \\
\text { LS2 }(\mu \mathrm{s} / \mathrm{cm})\end{array}$ & 389 & 560 & 456,7 & 63,06 & 0,138 & 480 & 673 & 549,4 & 66,91 & 0,122 & 389 & 673 & 506,6 & 78,82 & 0,156 \\
\hline ST-LS1 (mg/L) & 410 & 928 & 620,3 & 180,68 & 0,291 & 311 & 450 & 368,0 & 54,02 & 0,147 & 311 & 928 & 484,5 & 179,46 & 0,370 \\
\hline ST-LS2 (mg/L) & 388 & 655 & 497,7 & 98,50 & 0,198 & 346 & 412 & 383,3 & 21,68 & 0,057 & 346 & 655 & 436,1 & 88,32 & 0,203 \\
\hline STF-LS1 (mg/L) & 142 & 325 & 266,0 & 74,90 & 0,282 & 198 & 264 & 236,9 & 25,52 & 0,108 & 142 & 325 & 250,3 & 53,78 & 0,215 \\
\hline STF-LS2 (mg/L) & 277 & 330 & 298,3 & 19,80 & 0,066 & 220 & 278 & 254,6 & 20,43 & 0,080 & 220 & 330 & 274,8 & 29,79 & 0,108 \\
\hline STV-LS1 (mg/L) & 235 & 603 & 354,3 & 134,24 & 0,379 & 70 & 235 & 131,1 & 55,64 & 0,424 & 70 & 603 & 234,2 & 149,89 & 0,640 \\
\hline STV-LS2 (mg/L) & 75 & 372 & 199,3 & 111,12 & 0,557 & 114 & 147 & 128,7 & 10,98 & 0,085 & 75 & 372 & 161,3 & 80,92 & 0,502 \\
\hline SDT-LS1 (mg/L) & 403 & 922 & 610,0 & 180,32 & 0,296 & 305 & 442 & 359,7 & 53,99 & 0,150 & 305 & 922 & 475,2 & 178,52 & 0,376 \\
\hline SDT-LS2 (mg/L) & 374 & 645 & 482,7 & 100,87 & 0,209 & 330 & 397 & 365,4 & 22,21 & 0,061 & 330 & 645 & 419,5 & 90,48 & 0,216 \\
\hline SDF-LS1 (mg/L) & 141 & 324 & 264,0 & $\begin{array}{l}74,53 \\
\end{array}$ & 0,282 & 196 & 260 & 234,7 & 24,81 & 0,106 & 141 & 324 & 248,2 & 53,41 & 0,215 \\
\hline SDF-LS2 (mg/L) & 272 & 328 & 296,0 & 19,97 & 0,067 & 217 & 274 & 250,4 & 20,07 & 0,080 & 217 & 328 & 271,5 & 30,44 & 0,112 \\
\hline SDV-LS1 (mg/L) & 230 & 598 & 346,0 & 134,60 & 0,389 & 59 & 228 & 125,0 & 55,98 & 0,448 & 59 & 598 & 227,0 & 149,22 & 0,657 \\
\hline SDV-LS2 (mg/L) & 65 & 363 & 186,7 & 111,51 & 0,597 & 104 & 137 & 115,0 & 10,61 & 0,092 & 65 & 363 & 148,1 & 81,36 & 0,549 \\
\hline SST-LS1 (mg/L) & 6 & 18 & 10,3 & 5,61 & 0,543 & 6 & 15 & 8,3 & 3,15 & 0,380 & 6 & 18 & 9,2 & 4,38 & 0,475 \\
\hline SST-LS2 (mg/L) & 9 & 31 & 15,0 & 8,29 & 0,553 & 14 & 31 & 17,9 & 5,93 & 0,332 & 9 & 31 & 16,5 & 6,96 & 0,421 \\
\hline SSF-LS1 (mg/L) & 1 & 5 & 2,0 & 1,55 & 0,775 & 1 & 4 & 2,1 & 1,07 & 0,499 & 1 & 5 & 2,1 & 1,26 & 0,605 \\
\hline SSF-LS2 (mg/L) & 1 & 5 & 2,3 & 1,75 & 0,751 & 3 & 5 & 4,1 & 0,69 & 0,167 & 1 & 5 & 3,3 & 1,55 & 0,468 \\
\hline SSV-LS1 (mg/L) & 5 & 15 & 8,3 & 4,46 & 0,535 & 4 & 11 & 6,1 & 2,34 & 0,381 & 4 & 15 & 7,2 & 3,51 & 0,490 \\
\hline SSV-LS2 (mg/L) & 7 & 26 & 12,7 & 7,06 & 0,557 & 10 & 26 & 13,7 & 5,62 & 0,410 & 7 & 26 & 13,2 & 6,07 & 0,459 \\
\hline Turbidez-LS1 (UT) & 15 & 57 & 27,2 & 16,01 & 0,589 & 17 & 26 & 21,7 & 3,50 & 0,161 & 15 & 57 & 24,2 & 10,99 & 0,454 \\
\hline Turbidez-LS2 (UT) & 15 & 52 & 33,0 & 12,31 & 0,373 & 22 & 34 & 27,0 & 4,47 & 0,166 & 15 & 52 & 29,8 & 9,10 & 0,306 \\
\hline $\begin{array}{l}\text { Cor Aparente } \\
\text { LS1 (uH) }\end{array}$ & 33 & 61 & 41,3 & 10,56 & 0,255 & 30 & 46 & 39,3 & 6,55 & 0,167 & 30 & 61 & 40,2 & 8,31 & 0,207 \\
\hline $\begin{array}{l}\text { Cor Aparente } \\
\text { LS2 (uH) }\end{array}$ & 32 & 77 & 55,8 & 14,78 & 0,265 & 36 & 61 & 46,7 & 8,62 & 0,184 & 32 & 77 & 50,9 & 12,27 & 0,241 \\
\hline $\begin{array}{l}\text { Cloreto } \\
\text { LS1 (mg/L) }\end{array}$ & 39,2 & 45,0 & 41,0 & 2,15 & 0,053 & 39,1 & 52,6 & 44,7 & 4,51 & 0,101 & 39,1 & 52,6 & 43,0 & 3,96 & 0,092 \\
\hline Cloreto-LS2 (mg/L) & 37,3 & 45,0 & 41,6 & 2,76 & 0,066 & 40,0 & 51,7 & 44,4 & 4,09 & 0,092 & 37,3 & 51,7 & 43,1 & 3,68 & 0,085 \\
\hline Amônio-LS1 (mg/L) & 0,0 & 0,4 & 0,2 & 0,17 & 0,981 & 0,0 & 0,2 & 0,1 & 0,06 & 0,730 & 0,0 & 0,4 & 0,1 & 0,13 & 1,027 \\
\hline Amônio-LS2 (mg/L) & 0,0 & 4,0 & 1,2 & 1,64 & 1,367 & 0,0 & 0,4 & 0,1 & 0,12 & 0,916 & 0,0 & 4,0 & 0,6 & 1,20 & 1,916 \\
\hline Nitrato-LS1 (mg/L) & 7,7 & 17,4 & 13,1 & 3,82 & 0,291 & 4,7 & 12,1 & 8,8 & 2,57 & 0,293 & 4,7 & 17,4 & 10,8 & 3,81 & 0,353 \\
\hline Nitrato-LS2 (mg/L) & 8,1 & 20,4 & 12,4 & 5,28 & 0,427 & 7,8 & 12,1 & 9,3 & 1,41 & 0,151 & 7,8 & 20,4 & 10,7 & 3,88 & 0,362 \\
\hline Nitrito-LS1 (mg/L) & 0,2 & 0,5 & 0,4 & 0,12 & 0,307 & 0,1 & 0,3 & 0,2 & 0,06 & 0,284 & 0,1 & 0,5 & 0,3 & 0,12 & 0,431 \\
\hline Nitrito-LS2 (mg/L) & 0,3 & 0,5 & 0,4 & 0,09 & 0,237 & 0,4 & 0,5 & 0,4 & 0,03 & 0,067 & 0,3 & 0,5 & 0,4 & 0,07 & 0,162 \\
\hline NTK-LS1 (mg/L) & 2,5 & 5,9 & 4,2 & 2,38 & 0,566 & 4,7 & 5,7 & 5,1 & 0,30 & 0,059 & 2,5 & 5,9 & 4,9 & 0,97 & 0,197 \\
\hline NTK-LS2 (mg/L) & 4,2 & 5,0 & 4,6 & 0,59 & 0,129 & 4,2 & 12,6 & 9,3 & 2,55 & 0,274 & 4,2 & 12,6 & 8,3 & 3,03 & 0,367 \\
\hline $\begin{array}{l}\text { Fosforo total } \\
\text { LS1 (mg/L) }\end{array}$ & 3,8 & 5,5 & 4,7 & 0,61 & 0,130 & 4,5 & 5,3 & 5,0 & 0,25 & 0,051 & 3,8 & 5,5 & 4,9 & 0,45 & 0,094 \\
\hline $\begin{array}{l}\text { Fosforo total } \\
\text { LS2 (mg/L) }\end{array}$ & 4,6 & 6,4 & 5,8 & 0,64 & 0,110 & 5,9 & 6,7 & 6,4 & 0,30 & 0,046 & 4,6 & 6,7 & 6,1 & 0,57 & 0,093 \\
\hline
\end{tabular}

Tabela 2 - Valores mínimos, máximos e médios, desvios padrão e coeficientes de variação dos resultados das análises dos parâmetros físicos e químicos dos efluentes provenientes das Lagoas LS1 e LS2, durante o período de estudo. 


\begin{tabular}{|c|c|c|c|c|c|}
\hline \multirow[t]{2}{*}{ Parâmetro } & \multicolumn{2}{|c|}{$\begin{array}{c}\text { Teste de Normalidade } \\
\text { Shapiro Wilk com } \alpha=5 \%\end{array}$} & \multirow{2}{*}{$\begin{array}{c}\text { Teste de Variância } \\
\text { com } \alpha=5 \%\end{array}$} & \multirow{2}{*}{$\begin{array}{l}\text { Teste de Wilcoxon } \\
\text { com } \alpha=10 \%\end{array}$} & \multirow[t]{2}{*}{ Teste $\mathrm{t} \operatorname{com} \alpha=10 \%$} \\
\hline & LS 1 & LS 2 & & & \\
\hline $\mathrm{DQO}(\mathrm{mg} / \mathrm{L})$ & 0,187 & 0,307 & 0,260 & 0,113 & 0,098 \\
\hline $\mathrm{DBO}(\mathrm{mg} / \mathrm{L})$ & 0,280 & 0,223 & 0,531 & 0,117 & 0,132 \\
\hline $\mathrm{pH}$ & 0,128 & 0,010 & 0,790 & 0,582 & $\left({ }^{*}\right)$ \\
\hline Condutividade $(\mu \mathrm{s} / \mathrm{cm})$ & 0,715 & 0,720 & 0,989 & 0,898 & 0,914 \\
\hline ST (mg/L) & 0,040 & 0,009 & 0,021 & 0,918 & $\left({ }^{*}\right)$ \\
\hline STF (mg/L) & 0,724 & 0,999 & 0,051 & 0,166 & 0,164 \\
\hline STV (mg/L) & 0,077 & 0,005 & 0,042 & 0,427 & $\left({ }^{*}\right)$ \\
\hline $\mathrm{SDT}(\mathrm{mg} / \mathrm{L})$ & 0,035 & 0,008 & 0,026 & 0,762 & $\left({ }^{*}\right)$ \\
\hline SDF (mg/L) & 0,780 & 1,000 & 0,063 & 0,174 & 0,186 \\
\hline SDV (mg/L) & 0,082 & 0,002 & 0,045 & 0,305 & $(*)$ \\
\hline SST (mg/L) & 0,001 & 0,004 & 0,123 & 0,003 & $(*)$ \\
\hline SSF (mg/L) & 0,008 & 0,016 & 0,479 & 0,061 & $\left({ }^{*}\right)$ \\
\hline SSV (mg/L) & 0,002 & 0,003 & 0,069 & 0,003 & $(*)$ \\
\hline Turbidez (uT) & 0,001 & 0,435 & 0,523 & 0,040 & $\left({ }^{*}\right)$ \\
\hline Cor Aparente $(\mathrm{uH})$ & 0,094 & 0,880 & 0,191 & 0,024 & 0,016 \\
\hline Cloreto (mg/L) & 0,047 & 0,579 & 0,805 & 0,700 & $(*)$ \\
\hline Amônio (mg/L) & 0,015 & 0,000 & 0,000 & 0,341 & $\left({ }^{*}\right)$ \\
\hline Nitrato (mg/L) & 0,829 & 0,000 & 0,948 & 0,837 & $\left({ }^{*}\right)$ \\
\hline Nitrito (mg/L) & 0,023 & 0,068 & 0,037 & 0,042 & $\left({ }^{*}\right)$ \\
\hline NTK (mg/L) & 0,005 & 0,133 & 0,004 & 0,093 & $(*)$ \\
\hline Fósforo Total (mg/L) & 0,464 & 0,018 & 0,450 & 0,000 & $\left({ }^{*}\right)$ \\
\hline
\end{tabular}

(*) O Teste $t$ não foi utilizado devido ao teste de Shapiro Wilk ter rejeitado a normalidade com $\alpha=5 \%$

Tabela 3 - Resultados dos p-valores referentes aos testes de hipóteses realizados com todos os resultados obtidos no período de 90 dias (13 coletas) de operação da ETEP

\begin{tabular}{|c|c|c|c|c|c|}
\hline \multirow[t]{2}{*}{ Parâmetro } & \multicolumn{2}{|c|}{$\begin{array}{c}\text { Teste de Normalidade } \\
\text { Shapiro Wilk com } \alpha=5 \%\end{array}$} & \multirow{2}{*}{$\begin{array}{l}\text { Teste de Variância } \\
\text { com } \alpha=5 \%\end{array}$} & \multirow{2}{*}{$\begin{array}{l}\text { Teste de Wilcoxon } \\
\text { com } \alpha=10 \%\end{array}$} & \multirow[t]{2}{*}{ Teste $\mathrm{t} \operatorname{com} \alpha=10 \%$} \\
\hline & LS 1 & LS 2 & & & \\
\hline $\mathrm{DQO}(\mathrm{mg} / \mathrm{L})$ & 0,574 & 0,629 & 0,318 & 0,485 & 0,293 \\
\hline $\mathrm{DBO}(\mathrm{mg} / \mathrm{L})$ & 0,140 & 0,310 & 0,605 & 0,229 & 0,224 \\
\hline $\mathrm{pH}$ & 0,184 & 0,828 & 0,756 & 0,809 & 0,962 \\
\hline Condutividade $(\mu \mathrm{s} / \mathrm{cm})$ & 0,787 & 0,461 & 0,880 & 0,748 & 0,875 \\
\hline ST (mg/L) & 0,657 & 0,596 & 0,209 & 0,240 & 0,175 \\
\hline STF (mg/L) & 0,116 & 0,606 & 0,011 & 0,748 & 0,348 \\
\hline STV (mg/L) & 0,123 & 0,797 & 0,688 & 0,065 & 0,054 \\
\hline $\mathrm{SDT}(\mathrm{mg} / \mathrm{L})$ & 0,569 & 0,355 & 0,228 & 0,240 & 0,162 \\
\hline SDF (mg/L) & 0,137 & 0,852 & 0,012 & 0,688 & 0,351 \\
\hline SDV (mg/L) & 0,096 & 0,686 & 0,690 & 0,065 & 0,050 \\
\hline SST (mg/L) & 0,022 & 0,026 & 0,411 & 0,228 & $\left({ }^{*}\right)$ \\
\hline SSF (mg/L) & 0,010 & 0,059 & 0,795 & 0,931 & $(*)$ \\
\hline SSV (mg/L) & 0,024 & 0,043 & 0,335 & 0,147 & $(*)$ \\
\hline Turbidez (uT) & 0,066 & 0,949 & 0,579 & 0,470 & 0,495 \\
\hline Cor Aparente (uH) & 0,083 & 0,859 & 0,478 & 0,173 & 0,079 \\
\hline Cloreto (mg/L) & 0,100 & 0,917 & 0,598 & 0,570 & 0,667 \\
\hline Amônio (mg/L) & 0,256 & 0,045 & 0,000 & 0,423 & $(*)$ \\
\hline Nitrato (mg/L) & 0,392 & 0,046 & 0,496 & 0,937 & $(*)$ \\
\hline Nitrito (mg/L) & 0,035 & 0,388 & 0,595 & 0,872 & $(*)$ \\
\hline NTK (mg/L) & - & - & - & - & - \\
\hline Fósforo Total (mg/L) & 0,683 & 0,097 & 0,922 & 0,015 & 0,013 \\
\hline
\end{tabular}

(*) O Teste t não foi utilizado devido ao teste de Shapiro Wilk ter rejeitado a normalidade com $\alpha=5 \%$.

Tabela 4 - Resultados dos p-valores referentes aos testes de hipóteses realizados com os resultados obtidos até o $42^{\circ}$ dia $(6$ coletas) de operação da ETEP. 


\begin{tabular}{|c|c|c|c|c|c|}
\hline \multirow{2}{*}{ Parâmetro } & \multicolumn{2}{|c|}{$\begin{array}{c}\text { Teste de Normalidade } \\
\text { Shapiro Wilk com } \alpha=5 \%\end{array}$} & \multirow{2}{*}{$\begin{array}{l}\text { Teste de Variância } \\
\text { com } \alpha=5 \%\end{array}$} & \multirow{2}{*}{$\begin{array}{l}\text { Teste de Wilcoxon } \\
\text { com } \alpha=10 \%\end{array}$} & \multirow{2}{*}{ Teste t com $\alpha=10 \%$} \\
\hline & LS 1 & LS 2 & & & \\
\hline $\mathrm{DQO}(\mathrm{mg} / \mathrm{L})$ & 0,120 & 0,134 & 0,848 & 0,128 & 0,223 \\
\hline DBO (mg/L) & 0,733 & 0,776 & 0,624 & 0,442 & 0,444 \\
\hline $\mathrm{pH}$ & 0,873 & 0,024 & 0,059 & 0,192 & $\left({ }^{*}\right)$ \\
\hline Condutividade $(\mu \mathrm{s} / \mathrm{cm})$ & 0,155 & 0,411 & 0,978 & 1,000 & 0,969 \\
\hline ST (mg/L) & 0,335 & 0,748 & 0,043 & 0,383 & 0,507 \\
\hline STF (mg/L) & 0,382 & 0,566 & 0,603 & 0,179 & 0,177 \\
\hline STV (mg/L) & 0,152 & 0,942 & 0,001 & 0,224 & 0,913 \\
\hline $\mathrm{SDT}(\mathrm{mg} / \mathrm{L})$ & 0,260 & 0,986 & 0,048 & 0,535 & 0,802 \\
\hline SDF (mg/L) & 0,345 & 0,640 & 0,620 & 0,224 & 0,217 \\
\hline SDV (mg/L) & 0,224 & 0,081 & 0,001 & 0,522 & 0,658 \\
\hline SST (mg/L) & 0,012 & 0,001 & 0,149 & 0,005 & $(*)$ \\
\hline SSF (mg/L) & 0,294 & 0,099 & 0,311 & 0,007 & 0,001 \\
\hline SSV (mg/L) & 0,036 & 0,003 & 0,051 & 0,006 & $(*)$ \\
\hline Turbidez (uT) & 0,610 & 0,617 & 0,566 & 0,063 & 0,030 \\
\hline Cor Aparente (uH) & 0,220 & 0,657 & 0,522 & 0,141 & 0,094 \\
\hline Cloreto (mg/L) & 0,739 & 0,597 & 0,817 & 1,000 & 0,908 \\
\hline Amônio (mg/L) & 0,747 & 0,371 & 0,113 & 0,402 & 0,350 \\
\hline Nitrato $(\mathrm{mg} / \mathrm{L})$ & 0,906 & 0,197 & 0,171 & 0,898 & 0,621 \\
\hline Nitrito (mg/L) & 0,153 & 0,299 & 0,113 & 0,002 & 0,000 \\
\hline NTK (mg/L) & 0,549 & 0,1441 & 0,000 & 0,030 & 0,005 \\
\hline Fósforo Total (mg/L) & 0,552 & 0,430 & 0,708 & 0,001 & 0,000 \\
\hline
\end{tabular}

(*) O Teste $t$ não foi utilizado devido ao teste de Shapiro Wilk ter rejeitado a normalidade com $\alpha=5 \%$.

Tabela 5 - Resultados do p-valores referentes aos testes de hipóteses realizados com os resultados obtidos do $43^{\circ}$ ao $90^{\circ}$ dia $(7$ coletas) de operação da ETEP

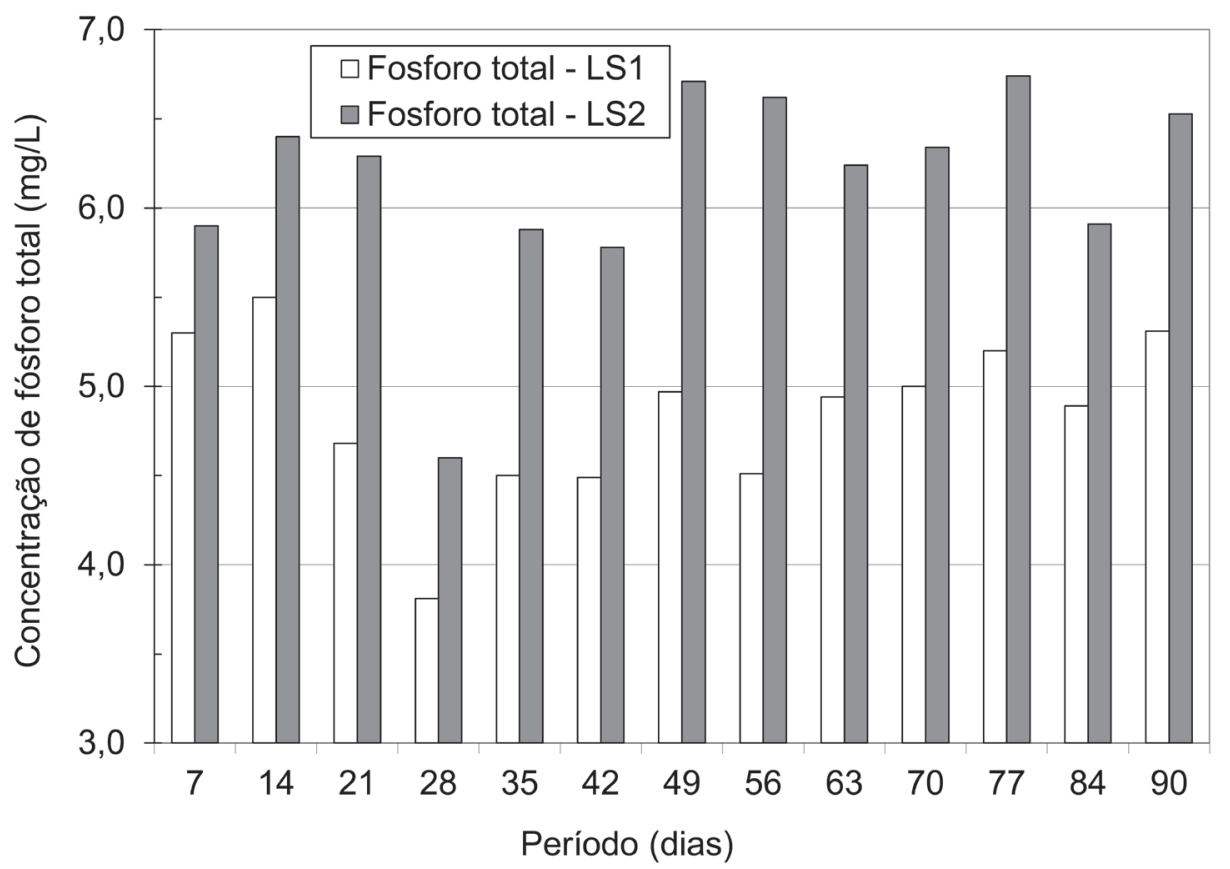

Figura 2 - Concentração de fósforo total nos efluentes das lagoas de sedimentação durante a operação da ETEP. 
3.1. Taxa de Consumo de Oxigênio e Caracterização do Licor Misto das Lagoas de Aeração

Para determinação da taxa de consumo de oxigênio (TCO) foram observado que as correlações das retas atingiram valores superiores a 99,2 $\%$, e as TCO, que são determinadas pelos coeficientes angulares das retas, foram superiores na LA2, que não recebeu RETA. O menor consumo de oxigênio foi observado na LA1, fato associado à presença de RETA, que interferiu de alguma forma, por inibição ou por diminuição da quantidade de microrganismos. A menor $\mathrm{TCO}_{\mathrm{a}}$ e a menor relação $\mathrm{TCO}_{\mathrm{a}} / \mathrm{SSV}$ na LA1, indica que o RETA promoveu redução tanto na quantidade de microrganismos como na TCO. Os valores da TCO e sua relação com SSV encontram-se na Tabela 6.

Na Tabela 6 pode ser observada que a quantidade de sólidos sedimentados presentes na LA1 foi superior à da lagoa LA2. No entanto, os ST, representados em sua maior totalidade pelos SDT, foram superiores na LA2. Em relação aos SST, a LA1 apresentou maior concentração na forma de SSF, ao contrário da LA2, que apresentou maior concentração de SSV. Dentre os metais pesquisados no licor misto das lagoas de aeração, o ferro foi o que apresentou maior divergência entre as

\begin{tabular}{|c|c|c|c|c|}
\hline \multirow{3}{*}{ Parâmetro } & \multicolumn{2}{|c|}{ Licor misto da LA1 (com RETA) } & \multicolumn{2}{|c|}{ Licor misto da LA2 (sem RETA) } \\
\hline & \multicolumn{4}{|c|}{ Tempo de operação (dia) } \\
\hline & 78 & 90 & 78 & 90 \\
\hline $\mathrm{TCO}_{\mathrm{a}}\left(\mathrm{TCO}_{\mathrm{a}} \mathrm{mg} / \mathrm{L} . \mathrm{h}\right)$ & 1,61 & 1,70 & 2,32 & 2,64 \\
\hline $\mathrm{TCO}_{\mathrm{a}} / \mathrm{SSV}\left(\mathrm{mgO}_{2} / \mathrm{L} . \mathrm{h}\right) /\left(\mathrm{mgSSV} / \mathrm{L}_{\text {licor }}\right)$ & $8,9 \times 10^{-3}$ & $9,3 \times 10^{-3}$ & $10,8 \times 10^{-3}$ & $11,2 \times 10^{-3}$ \\
\hline DQO (mg/L) & 361,4 & 387,9 & 526,2 & 571,5 \\
\hline $\mathrm{pH}$ & 7,1 & 7,2 & 7,1 & 7,3 \\
\hline $\mathrm{OD}(\mathrm{mg} / \mathrm{L})$ & 3,10 & 3,20 & 2,75 & 2,90 \\
\hline Condutividade $(\mu \mathrm{s} / \mathrm{cm})$ & 525 & 545 & 540 & 536 \\
\hline Sólidos Totais (mg/L) & 790,0 & 856,0 & 950 & 1046,0 \\
\hline Sólidos Fixos (mg/L) & - & 484,0 & - & 520,0 \\
\hline Sólidos Voláteis (mg/L) & - & 372,0 & - & 526,0 \\
\hline Sólidos Suspensos Totais (mg/L) & 374,2 & 393,1 & 401,5 & 396,5 \\
\hline Sólidos Suspensos Fixos (mg/L) & - & 209,8 & - & 161,1 \\
\hline Sólidos Suspensos Voláteis (mg/L) & - & 183,3 & - & 235,4 \\
\hline Sólidos Dissolvidos Totais (mg/L) & 415,8 & 462,9 & 548,5 & 649,5 \\
\hline Sólidos Dissolvidos Fixos (mg/L) & - & 274,2 & - & 358,9 \\
\hline Sólidos Dissolvidos Voláteis (mg/L) & - & 188,7 & - & 290,6 \\
\hline Sol. Sed. em Cone Imhoff (mL/L) - 1hora & 15,5 & 16,0 & 13,0 & 13,5 \\
\hline Mn (mg Mn/g SST) & - & 1,376 & - & 0,931 \\
\hline $\mathrm{Fe}$ (mg Fe/g SST) & - & 99,568 & - & 35,914 \\
\hline $\mathrm{Ni}$ (mg Ni/g SST) & - & 1,068 & - & 1,354 \\
\hline Zn (mg Zn/g SST) & - & 1,068 & - & 1,077 \\
\hline $\mathrm{Pb}$ (mg Pb/g SST) & - & 0,483 & - & 0,641 \\
\hline $\mathrm{Cd}$ (mg Cd/g SST) & - & $<0,001$ & - & $<0,001$ \\
\hline $\mathrm{Cu}$ (mg Cu/g SST) & - & 0,333 & - & 0,390 \\
\hline $\mathrm{Cr}(\mathrm{mg} \mathrm{Cr} / \mathrm{g} \mathrm{SST})$ & - & 1,913 & - & 2,087 \\
\hline $\mathrm{Co}(\mathrm{mg} \mathrm{Co} / \mathrm{g} \mathrm{SST})$ & - & 0,168 & - & 0,128 \\
\hline
\end{tabular}

Tabela 6 - Valores da TCOa nos licores mistos das LA1 e LA2 e as relações TCOa/SSV, e resultados dos parâmetros pesquisados 
duas lagoas, sendo encontrado em maior concentração na LA1. Isto ocorreu devido ao RETA, adicionado no módulo 1, apresentar concentração de 340,8 mg Fe/ $\mathrm{L}_{\text {residuo. }}$ Em relação aos demais metais houve diferenças, mas nenhuma tão expressiva.

\subsection{Ensaio de Sedimentação}

Devido à pequena concentração de sólidos no licor misto das lagoas de aeração, foi necessária a utilização do Cone Imnhoff para poder avaliar a sedimentação do lodo. Pode ser observado na Figura 2 que o volume de sólidos sedimentáveis atingiu seu pico após 7 minutos de sedimentação no licor misto da LA1 e após 10 minutos no licor da LA2, evidenciando que seriam necessários menores tempos de sedimentação para a lagoa que recebeu RETA. Após este tempo, o material começou a sofrer adensamento, atingindo, no final do ensaio, $15,5 \mathrm{~mL}$ e $13,0 \mathrm{~mL}$, respectivamente. Portanto, o volume de sólidos sedimentados, decorridos 60 minutos, foi maior para o material que recebeu RETA, sendo 19,2\% maior que na LS2.

\subsection{Exame Microscópico}

O exame microscópico foi realizado com o licor misto das duas lagoas de aeração após 30 dias de operação das ETEP sem receber RETA. No $30^{\circ}$ dia foi constada a presença de organismos tais como Ciliados livre-natantes, Nematóides e ciliados pedunculados, indicadores do funcionamento adequado dos dois módulos de tratamento da ETEP. Alguns organismos, como o Rizopode, semelhante à Ameba, indicaram que o lodo era jovem caracterizando início de operação. Foi detectada a presença do ciliado livre Aspidisca sp, indicativo da nitrificação. A presença de Anelídeos do gênero Aelosoma se deu em quantidades normais, indicando boas condições de oxigenação. Caso fossem encontradas grandes quantidades desse organismo, haveria indícios de excesso de oxigênio, o que seria indesejável para o funcionamento da ETEP. O exame microscópico do material coletado indicou grande variedade de organismos tais como rotíferos, anelídeos e nematoides mostrando boas condições de depuração das duas ETEP, com características de lodo jovem, em início de operação.

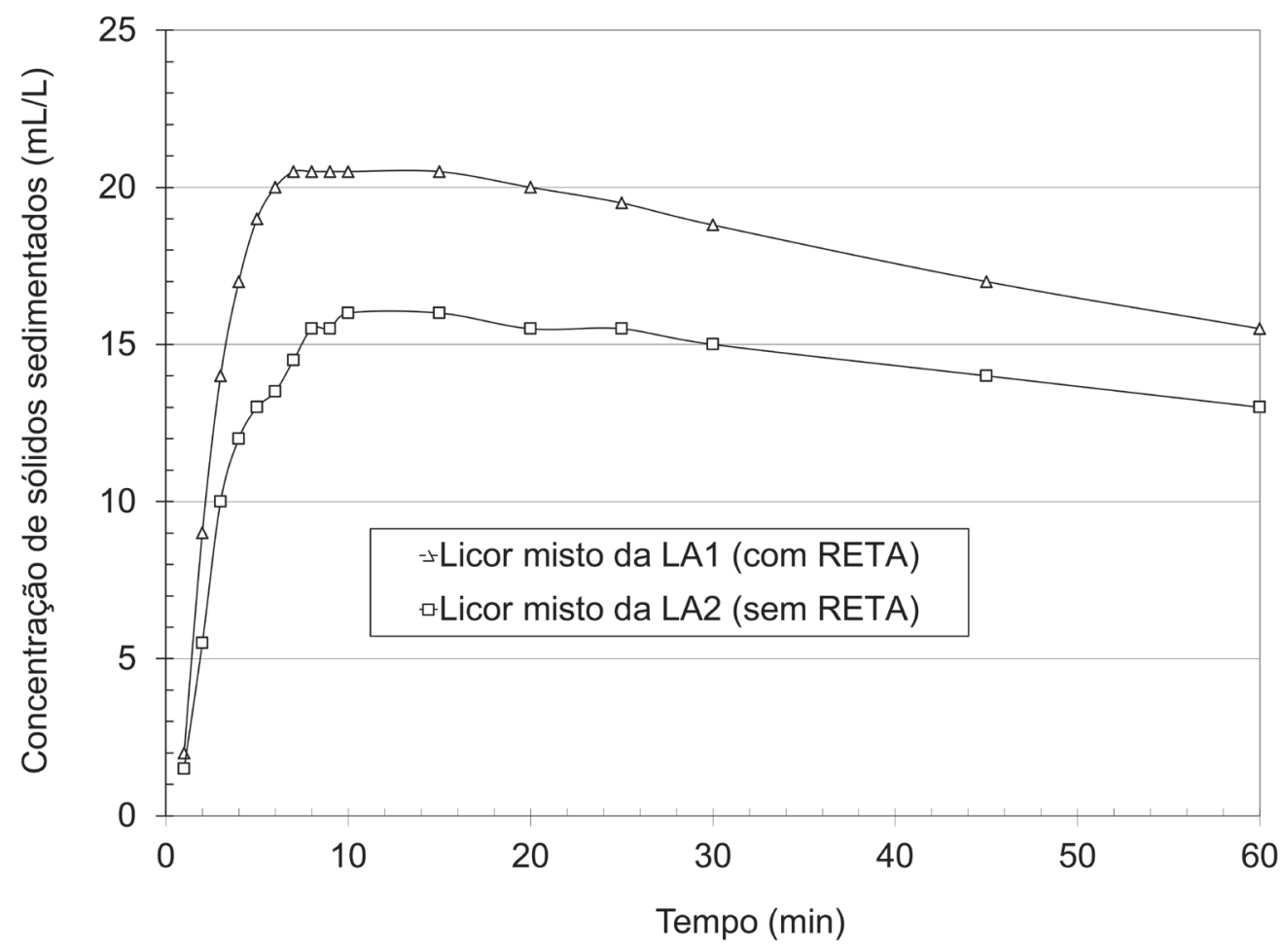

Figura 3 - Concentração de sólidos sedimentados nos licores mistos das lagoas de aeração, realizado em Cone Imnhoff em função do tempo. 
No $90^{\circ}$ dia de operação, com aplicação de RETA na lagoa LA1, foi realizado novo exame microscópico do licor misto das lagoas de aeração. O licor misto da LA1 (que recebeu RETA) não apresentou interferências negativas aparentes na sua microfauna. $\mathrm{O}$ aspecto deste material ao microscópio foi de flocos bem formados com presença de ciliados pedunculados e, nos espaços entre flocos, de ciliados livre-natantes e rotíferos, com presença também de nematóides que são indicativos de boa depuração. Foram encontrados anelídeos do gênero Aelosoma em condições normais. Uma característica deste material foi a ausência de bactérias filamentosas e bactérias do gênero Spirillum que estavam presentes no licor misto da LA2 que não recebeu RETA. A quantidade de organismos presentes no licor misto da LA1 (com RETA) foi ligeiramente superior em relação ao licor misto da LA2 (sem RETA).

\subsection{Caracterização do Lodo das Lagoas de Sedi- mentação}

Visando à caracterização e o conhecimento do volume de lodo gerado nos dois módulos da ETEP foram realizadas suas caracterizações no final da operação. Após a retirada do sobrenadante com auxílio de uma bomba, os lodos das lagoas de sedimentação foram coletados e caracterizados (ver Tabela 7). Os ST do lodo da LS1 apresentaram-se com menor concentração, mas em maior volume. A massa total de sólidos foi de 49,47 g (30,29 g/L x 1,6 L lodo), e 43,05 g (35,3 g/L x 1,22 L lodo), respectivamente, ou seja, a LS1, que recebeu RETA, apresentou uma maior quantidade de ST. Estes, foram representados por $49,81 \%(24,64 \mathrm{~g})$ de STF e $50,19 \%$ (24,83 g) de STV ao contrário do lodo da LS2, proveniente da ETEP que não recebeu RETA, onde $41,17 \%$ (17,73 g) foram na forma de STF e

\begin{tabular}{l|c|c}
\multicolumn{1}{c|}{ Parâmetro } & Lagoa LS1 & Lagoa LS2 \\
\hline DQO (g/L) & 23,2 & 30,9 \\
\hline pH & 7,1 & 7,0 \\
\hline Sólidos Totais (g/L) & 30,9 & 35,3 \\
\hline Sólidos Fixos (g/L) & 15,4 & 14,5 \\
\hline Sólidos Voláteis (g/L) & 15,5 & 20,8 \\
\hline Sólidos Suspensos Totais (g/L) & 28,2 & 32,0 \\
\hline Sólidos Suspensos Fixos (g/L) & 13,0 & 11,6 \\
\hline Sólidos Suspensos Voláteis (g/L) & 15,2 & 20,4 \\
\hline Sólidos Dissolvidos Totais (g/L) & 2,7 & 3,3 \\
\hline Sólidos Dissolvidos Fixos (g/L) & 2,4 & 2,9 \\
\hline Sólidos Dissolvidos Voláteis (g/L) & 0,3 & 0,4 \\
\hline Resistência Específica (x 10 ${ }^{13}$ m/Kg) & 1,42 & 4,40 \\
\hline Volume total de Lodo (L/15L) & 1,60 & 1,22 \\
\hline Massa total de sólidos (g) & 49,5 & 43,5 \\
\hline Manganês (mg Mn / Kg SST) & 924,1 & 369 \\
\hline Ferro (mg Fe / Kg SST) & 998237 & 36041 \\
\hline Níquel (mg Ni / Kg SST) & 221 & 442 \\
\hline Zinco (mg Zn / Kg SST) & 1237 & 1786 \\
\hline Chumbo (mg Pb / Kg SST) & 128 & 143 \\
\hline Cádmio (mg Cd / Kg SST) & 5,1 & 5,3 \\
\hline Cobre (mg Cu / Kg SST) & 292 & 19,7 \\
\hline Cromo (mg Cr / Kg SST) & 1169 & 21,2 \\
\hline Cobalto (mg Co / Kg SST) & & \\
\hline
\end{tabular}

Obs.: LS1 - Proveniente do módulo da ETEP que recebeu RETA; LS2 - proveniente do módulo da ETEP que não recebeu RETA

Tabela 7 - Caracterização dos lodos obtidos nas lagoas de sedimentação após o término da operação das ETEP 
$58,83 \%$ (25,33 g) na forma de STV.

A caracterização do lodo permitiu verificar que a massa de DQO encontrada no lodo foi de 37,14 g na LS1 e 37,7 g na LS2, valores praticamente iguais. Com relação aos metais pesquisados, observa-se que o Ferro e Manganês foram os que apresentaram maiores divergências. A massa do ferro no lodo da LS1 foi de $4504 \mathrm{mg}$ (2815 mg/L x 1,6 L lodo), contra 1407 mg no lodo da LS2 (1153 mg/L x 1,22 L lodo), representando $220,11 \%$ a mais deste metal. Para o manganês, o aumento foi de $189,51 \%$. Estes valores são explicados pela grande quantidade desses metais no RETA, pois é utilizado o cloreto férrico como coagulante na ETA em valores de $\mathrm{pH}$ de coagulação entre 8,5 e 9,0, o que favorece a formação de precipitados de metais presentes na água bruta.

\subsubsection{Resistência Específica do lodo}

Os ensaios para determinação da resistência específica foram realizados com os lodos provenientes das lagoas LS1 e LS2 após o en- cerramento da operação da ETEP. O valor de resistência específica determinado no lodo da LS1 foi de $1,42 \times 10^{13} \mathrm{~m} / \mathrm{Kg}$, sendo $67,73 \%$ menor que a resistência específica do LS2, que foi de $4,40 \times 10^{13} \mathrm{~m} / \mathrm{Kg}$. Certamente esse fato se deve à presença de espécies hidrolisadas de ferro (e possivelmente de outros metais) que funcionaram como condicionantes que favoreceram a filtração de lodo da lagoa LS1 em tempo menor, resultando, consequentemente, menor resistência específica.

\subsubsection{Desaguamento do lodo por centrifugação}

Os valores de $\mathrm{SST}_{\mathrm{r}}$ na torta, após 30 minutos de centrifugação foram de 128,2 g/L e 128,0 g/L, para os lodos da LS1 e LS2, respectivamente, sendo que para atingir esta concentração foram necessários 2,0 g de polieletrólito/Kg de SST para o lodo da lagoa LS1 que recebeu RETA e, de 3,5 g de polieletrólito/Kg de SST para o lodo da LS2, evidenciando que o uso de RETA no módulo LA1-LS1 da ETEP favoreceu o desaguamento me-

\begin{tabular}{|c|c|c|c|c|c|c|c|c|c|c|}
\hline \multirow{3}{*}{$\begin{array}{r}\text { Parâmetro } \\
\text { g poli/Kg SST }\end{array}$} & \multicolumn{10}{|c|}{ Sobrenadantes do centrifugado } \\
\hline & \multicolumn{5}{|c|}{$\begin{array}{c}\text { Sobrenadante após da centrifugação do lodo prove- } \\
\text { niente da LS1 (com RETA) }\end{array}$} & \multicolumn{5}{|c|}{$\begin{array}{c}\text { Sobrenadante após da centrifugação do lodo prove } \\
\text { niente da LS2 (sem RETA) }\end{array}$} \\
\hline & 0,0 & 1,0 & 1,5 & 2,0 & 2,5 & 0,0 & 2,0 & 3,0 & 3,5 & 4,0 \\
\hline Turbidez (UT) & 64 & 36 & 22 & 21 & 27 & 124 & 72 & 60 & 51 & 60 \\
\hline Cor $(\mathrm{uH})$ & 332 & 202 & 130 & 110 & 164 & 688 & 352 & 270 & 152 & 280 \\
\hline DQO (mg/L) & 233,7 & 292,3 & 269,4 & 186,7 & 248,3 & 400,6 & 397,7 & 420,6 & 224,9 & 261,1 \\
\hline
\end{tabular}

Tabela 8 - Caracterização dos sobrenadantes obtidos no ensaio de centrifugação

\begin{tabular}{l|c|c}
\multirow{2}{*}{ Parâmetro } & \multicolumn{2}{|c}{ Concentração de Metais em Função da Massa de Sólidos Totais } \\
\cline { 2 - 3 } & Lodo LS1 com 2,0 g poli/kg SST & Lodo LS2 com 3,5 g poli/kg SST \\
\hline $\mathrm{Mn}(\mathrm{g} \mathrm{Mn} / \mathrm{kg} \mathrm{ST})$ & 0,726 & 0,348 \\
\hline $\mathrm{Fe}(\mathrm{g} \mathrm{Fe} / \mathrm{kg} \mathrm{ST})$ & 81,817 & 37,058 \\
\hline $\mathrm{Ni}(\mathrm{g} \mathrm{Ni} / \mathrm{kg} \mathrm{ST})$ & 0,183 & 0,422 \\
\hline $\mathrm{Zn}(\mathrm{g} \mathrm{Zn} / \mathrm{kg} \mathrm{ST})$ & 1,184 & 1,263 \\
\hline $\mathrm{Pb}(\mathrm{g} \mathrm{Pb} / \mathrm{kg} \mathrm{ST})$ & 0,099 & 0,124 \\
\hline $\mathrm{Cd}(\mathrm{g} \mathrm{Cd} / \mathrm{kg} \mathrm{ST})$ & 0,003 & 0,004 \\
\hline $\mathrm{Cu}(\mathrm{g} \mathrm{Cu} / \mathrm{kg} \mathrm{ST})$ & 0,246 & 0,340 \\
\hline $\mathrm{Cr}(\mathrm{g} \mathrm{Cr} / \mathrm{kg} \mathrm{ST})$ & 0,660 & 1,382 \\
\hline $\mathrm{Co}(\mathrm{g} \mathrm{Co} / \mathrm{kg} \mathrm{ST})$ & 0,024 & 0,023
\end{tabular}

Tabela 9 - Concentração de metais nas tortas de lodo obtidas nos melhores ensaios de centrifugação com os lodos coletados nas lagoas LS1 e LS2. 
cânico do lodo.

Nota-se, na Tabela 8, que os parâmetros pesquisados foram menores para os sobrenadantes dos centrifugados provenientes da lagoa LS1 do módulo que recebeu RETA, apresentando melhor qualidade.

Com relação à presença de metais nas tortas de lodo obtidas nos melhores ensaios de centri- fugação, a Tabela 9 mostra que as concentrações, em função da massa de sólidos totais, de zinco, chumbo, cádmio, cobre e cobalto resultaram praticamente iguais com os lodos provenientes das lagoas de sedimentação dos dois módulos. As concentrações de ferro e manganês na torta 1 foram superiores às da torta 2 e as de níquel e cromo resultaram menores.

\begin{tabular}{|c|c|c|c|c|c|c|}
\hline \multirow{3}{*}{ Poluente (mg/L) } & \multicolumn{3}{|c|}{ Lixiviação } & \multicolumn{3}{|c|}{ Solubilização } \\
\hline & \multirow{2}{*}{$\begin{array}{c}\text { LMP } \\
(\mathrm{mg} / \mathrm{L})\end{array}$} & \multicolumn{2}{|c|}{ Resultado (mg/L) } & \multirow{2}{*}{$\begin{array}{l}\text { LMP } \\
\text { LS1 }\end{array}$} & \multicolumn{2}{|c|}{ Resultado (mg/L) } \\
\hline & & LS1 & LS2 & & LS1 & LS2 \\
\hline Arsênio & 5,0 & $<0,0001$ & $<0,0001$ & 0,05 & $<0,0001$ & $<0,0001$ \\
\hline Bário & 100,0 & $<0,05$ & $<0,05$ & 1,0 & $<0,05$ & $<0,05$ \\
\hline Cádmio & 0,05 & 0,032 & 0,032 & 0,005 & $<0,05$ & $<0,05$ \\
\hline Chumbo & 5,0 & 0,127 & 0,136 & 0,05 & 0,496 & 0,476 \\
\hline Cianeto & NN & NN & NN & 0,1 & $<0,001$ & $<0,001$ \\
\hline Fenol & NN & NN & NN & 0,001 & NR & NR \\
\hline Nitrato & NN & NN & NN & 10,0 & 8,28 & 5,25 \\
\hline Cromo Total & 5,0 & 0,032 & 0,051 & 0,05 & 0,184 & 0,188 \\
\hline Fluoreto & 150,0 & 0,25 & 1,34 & 1,5 & 0,32 & $<0,1$ \\
\hline Mercúrio & 0,1 & 0,019 & 0,020 & 0,001 & 0,052 & 0,0224 \\
\hline Prata & 5,0 & $<0,05$ & $<0,05$ & 0,05 & $<0,05$ & $<0,05$ \\
\hline Selênio & 1,0 & $<0,05$ & $<0,05$ & 0,01 & $<0,05$ & $<0,05$ \\
\hline Aldrin & 0,003 & 0,00001 & ND & $3,0 \times 10^{-5}$ & ND & ND \\
\hline DDT & 0,1 & ND & ND & $1,0 \times 10^{-4}$ & ND & ND \\
\hline Dieldrin & 0,003 & ND & ND & $3,0 \times 10^{-4}$ & ND & ND \\
\hline Endrin & 0,02 & ND & ND & $2,0 \times 10^{-4}$ & ND & ND \\
\hline Epóxi-heptacloro & 0,01 & ND & ND & $1,0 \times 10^{-4}$ & ND & ND \\
\hline Heptacloro & 0,01 & ND & ND & $1,0 \times 10^{-4}$ & ND & ND \\
\hline Hexaclorobenzeno & 0,001 & ND & ND & $1,0 \times 10^{-4}$ & ND & ND \\
\hline Lindano & 0,3 & ND & ND & $3,0 \times 10^{-4}$ & ND & ND \\
\hline Pentaclorofenol & 1,0 & 0,00002 & 0,000005 & 0,01 & ND & ND \\
\hline Alumínio & NN & NN & NN & 0,2 & 0,008 & 0,032 \\
\hline Cloreto & NN & NN & NN & 250,0 & 96,0 & 76,0 \\
\hline Cobre & NN & NN & NN & 1,0 & 0,112 & 0,116 \\
\hline Dureza & NN & NN & NN & 500,0 & 320,0 & 280,0 \\
\hline Ferro & NN & NN & NN & 0,3 & 4,812 & 4,712 \\
\hline Manganês & NN & NN & NN & 0,1 & 0,768 & 0,100 \\
\hline Sódio & NN & NN & NN & 200,0 & 200,0 & 180,0 \\
\hline Surfactantes & NN & NN & NN & 0,2 & NR & NR \\
\hline Sulfato & NN & NN & NN & 400,0 & 120,0 & 140,0 \\
\hline Zinco & NN & NN & NN & 5,0 & 0,54 & 4,98 \\
\hline
\end{tabular}

LMP: limite máximo permitido; ND: não detectado; NR: não realizado; NN: não necessário.

Tabela 10 - Resultados dos poluentes pesquisados nos extratos do lixiviado e do solubilizado das tortas obtidas nos ensaios de centrifugação com os lodos das LS1 e LS2, e seus limites máximos permitidos, de acordo com a NBR 10004/1987 Anexo G Listagem no 7 - Concentração - limite máximo no extrato obtido no teste de lixiviação e NBR 10004/1987 Anexo H Listagem no 8 - Padrões para o teste de solubilização. 


\section{Ensaios de Lixiviação e Solubilização das Tortas de Lodo}

Os resultados dos poluentes pesquisados nos extratos dos ensaios de lixiviação e solubilização encontram-se na Tabela 10, juntamente com seus limites máximos permitidos. Observa-se, no ensaio de solubilização, que chumbo, cromo total, mercúrio e ferro resultaram com concentrações superiores aos limites nos dois extratos, enquanto que apenas manganês apresentou concentração superior ao limite no solubilizado da torta de lodo da lagoa de sedimentação LS1. Os demais parâmetros pesquisados atenderam aos limites estabelecidos pela NBR 10004 (ABNT, 2004). Os resultados obtidos nos ensaios de lixiviação atenderam aos limites da referida norma.

\section{Conclusões}

O presente trabalho permitiu concluir que:

- O efluente final produzido pelo módulo de lagoa de aeração e lagoa de sedimentação que recebeu RETA apresentou melhor qualidade em termos de DQO, SST, turbidez, cor aparente, nitrito, NTK e fósforo total. As demais características pesquisadas não apresentaram diferenças significativas.

- No exame microscópico não houve influências negativas no licor misto da lagoa de aeração do módulo 1 que recebeu RETA. Neste material não foram encontradas bactérias filamentosas, as quais foram observadas no licor misto da lagoa de aeração que não recebeu RETA. Provavelmente a presença de RETA no módulo 1 inibiu o crescimento de bactérias filamentosas.

- Apesar da TCO ter sido menor na LA1 (que recebeu RETA) torna-se atrativa sua disposição na ETE, pois além de reduzir a quantidade de bactérias filamentosas no licor misto, houve maior eficiência na remoção de quase todos os parâmetros pesquisados no efluente final.

- Houve maior geração de lodo na lagoa de sedimentação do módulo que recebeu RETA e a quantidade de sólidos sedimentáveis nela foi, em média, 5,3 \% maior que na LA2. Essa diferença é relativamente pequena para ser considerada negativa ao método de disposição de RETA em ETE.

- Os valores de resistência específica e os ensaios de centrifugação evidenciaram uma melhor eficiência de desaguamento do lodo sedimentado na lagoa de sedimentação do módulo que recebeu RETA, gerando uma economia no consumo de polímero para desaguamento e resultando tortas com praticamente a mesma concentração de sólidos.

- Com relação aos ensaios de lixiviação e solubilização das tortas obtidas nos ensaios de centrifugação, foi constatado que apenas no ensaio de solubilização encontrou-se manganês com concentração superior ao limite máximo permitido.

\section{Refeferências}

ABNT - ASSOCIAÇÃO BRASILEIRA DE NORMAS TÉCNICAS. (2004) NBR 10004: Resíduos sólidos. Rio de Janeiro.

APHA; AWWA; WPCF. (2005) Standard Methods for the Examination of Water and Wastewater. 21 ed. Washington, D.C.

ACHON, C.L.; BARROSO, M.M.; CORDEIRO, J.S. (2008) Leito de drenagem: sistema natural para redução de volume de lodo de estação de tratamento de água. Revista Engenharia Sanitária e Ambiental, Rio de Janeiro, v. 13, n.1, jan/mar, p.54-62.

BOTERO, W. G.; SANTOS, A.; OLIVEIRA, L. C.; ROCHA, J. C. (2009) Caracterização de lodo gerado em Estações de Tratamento de água: perspectivas de aplicação agrícola. Revista Química Nova, v. 32, n. 8, p.2018-2022.

BUSSAB, W.O.; MORETTIN, P (2002). Estatística Básica. São Paulo, Ed. Saraiva, 5a edição.

CARVALHO, E.H. (2000) Disposição de resíduos gerados nas estações de tratamento de água em estações de tratamento de esgoto com decantação primária. São Carlos. Tese (Doutorado) - Escola de Engenharia de São Carlos, Universidade de São Paulo, 199p.

CHAO, I. R. S.; YABROUDI, S. C.; MORITA, D. M. (2011) Phosphorus removal from stabilization lagoon effluents using water treatment plant sludge. Interciencia, v.36, n.10, p. 774-778, out.

DI BERNARDO, L.; DANTAS, A.D.B; VOLTAN, P.E.N. (2011) Tratabilidade de água e dos resíduos gerados em estações de tratamento de água. São Carlos, Ed. LDiBe, 454p.

DAYTON, E. A. BASTA, N. T. (2001) Characterization of drinking water treatment residuals for use as a soil substitute. Water Environment Research, vo.73, n.1, p.52-57, jan-feb.

FERREIRA FILHO, S.S.; WAELKENS, B.E. (2009) Minimização da produção de lodo no tratamento de 
águas de abastecimento mediante uso do cloreto de polialumínio e sua disposição em estações de tratamento de esgotos. Revista Engenharia Sanitária e Ambiental, Rio de Janeiro, v. 14, n.3, jul/set, p. 317-326.

FIGUEIREDO NETO, A.; SCALIZE, P.S.; ALBUQUERQUE, A. (2012) Use of sludge treatment plant water in the production of tree seedlings with occurrence in the cerrado biome. In: 4th Internacional Conference on Engineering for Waste and Biomass Valorisation, Porto - Portugal, 2012. v. 3. p. 874-879.

JANUÁRIO, J.F; FERREIRA FILHO, S.S. (2007) Planejamento e aspectos ambientais envolvidos na disposição final de lodos das estações de tratamento de água da regi]ao metropolitana de São Paulo. Revista Engenharia Sanitária e Ambiental, Rio de Janeiro, v. 12, n.2, abr/jun, p.117-126.

MOREIRA, R.C.A.; GUIMARÃES, E.M.; BOAVENTURA, G.R; MOMESSO, A.M.; LIMA, G.L. (2009) Estudo geoquímico da disposição de lodo de estação de tratamento de água em área degradada. Revista Química Nova, São Paulo, v.32, n.8, p.2085-2093.

OH, T.K, NAKAJI, K., CHIKUSHI, J., PARK, S.G. (2010). Effects of the Application of Water Treatment Sludge on Growth of Lettuce (Lactuca sativa L.) and Changes in Soil Properties. Journal of the Faculty of Agriculture, Kyushu Univ., vol.55, n.1, p.15-20.

OLIVEIRA, E.M.S.; HOLANDA, J.N.F. (2008) Influência da adição de resíduo (lodo) de estação de tratamento de águas nas propriedades e microestrutura de cerâmica vermelha. Revista Cerâmica. São Paulo, vol.54, n.330, pp.167-173, jun.

REALI, M.A.P.; PATRIZZI, L.J; CORDEIRO, J.S. (1999) Desidratação de lodo por centrifugação. In: REALI, M.P., coord. Noções gerais de tratamento e disposição final de lodos de estações de tratamento de água. Rio de Janeiro, ABES, cap. 4, p. 85-106.

SCALIZE, P.S. (2003) Disposição de resíduos gerados em estações de tratamento de água em estações de tratamento de esgotos. São Carlos. Tese (Doutorado) - Escola de Engenharia de São Carlos, Universidade de São Paulo, 146p.

SCALIZE, P.S.; DI BERNARDO, L. (2011) Disposição de resíduos gerados em ETAs no decantador primário de ETEs. Revista Hydro (São Paulo), v. maio, p. $50-54$.
TEIXEIRA, S.T.; MELO, W.J.; SILVA, E.T. (2005) Aplicação de lodo da estação de tratamento de água em solo degradado. Pesquisa Agropecuária Brasileira, Brasília, v.40, n.1, p.91-94, jan.

TEIXEIRA,S.R.; SOUZA,S.A.; SOUZA, N.R.; ALÉSSIO, P.; SANTOS, G.T.A (2006) Efeito da adição de lodo de estação de tratamento de água (ETA) nas propriedades de material cerâmico estrutural. Revista Cerâmica, São Paulo: SP, v. 52, n.323, set/dez, p. 215-220.

TEIXEIRA, S.T.; MELO, W.J.; SILVA, E.T (2007) Plant nutrients in a degraded soil treated with water treatment sludge and cultivated with grasses and leguminous plants. Soil Biology \& Biochemistry, v.39, p.1348-1354.

TARTARI, R.; MÓDENES, A.N.; PIANARO, S.A.; DIAZ-MORA, N. (2011) Lodo gerado na estação de tratamento de água Tamanduá, Foz do Iguaçu, PR, como aditivo em argilas para cerâmica vermelha. Parte I: Caracterização do lodo e de argilas do terceiro planalto paranaense. Revista Cerâmica, São Paulo, vol.57, n. 343, p. 288-293. Set.

VON SPERLING, M. (1997). Princípios do tratamento biológico de águas residuárias. In: "Lodos Ativados", Departamento de Engenharia Sanitária e Ambiental; Universidade Federal de Minas Gerais, v.4, 415 p., Belo Horizonte, MG.

\section{SOBRE OS AUTORES}

\section{Paulo Sergio Scalize}

Engenheiro Civil e Biomédico, Mestre e Doutor em Hidráulica e Saneamento pela EESC-USP. Professor Ajunto da Escola de Engenharia Civil da Universidade Federal de Goiás (EEC-UFG). Luiz Di Bernardo

Engenheiro Civil. Doutor em Engenharia Civil. Professor Titular do Departamento de Hidráulica e Saneamento da Escola de Engenharia de São Carlos -

Universidade de São Paulo (USP) - São Carlos (SP), Brasil

Lorena Acelina Soares

Química Agroindustrial, formada pelo Instituto Federal de Educação, Ciência e Tecnologia de Goiás e Mestre em Engenharia do Meio Ambiente pela Universidade Federal de Goiás. Luis Rodrigo Fernandes Baumann

Graduado em Matemática pela Universidade Federal de Goiás. Mestre em matemática pela Universidade Federal de Goiás. Doutor em Estatística pela Universidade de São Paulo. Professor do Instituto de Matemática e Estatística da Universidade Federal de Goiás.

*Endereço: Escola de Engenharia Civil, Universidade Federal de Goiás, Campus Colemar Natal e Silva, Bloco A, sala 14. Avenida Universitária, 1488, CEP 74605-220 - Goiânia - GO - Brasil - Tel: +55 (62) 3209-6257.

E-mail: pscalize.ufg@gmail.com 


\section{O site da Revista DAE está repleto de novidades}

Assine gratuitamente o boletim
eletrônico, é só se cadastrar!

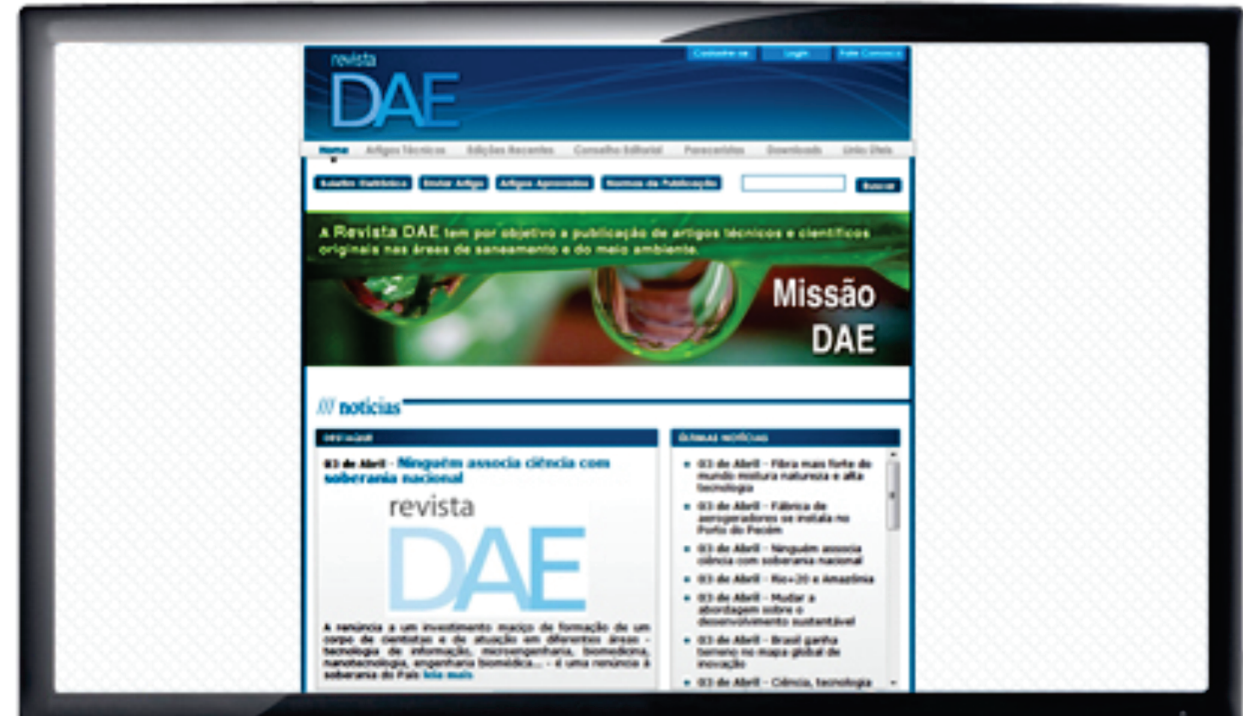

No site você pode baixar o conteúdo completo da Revista.

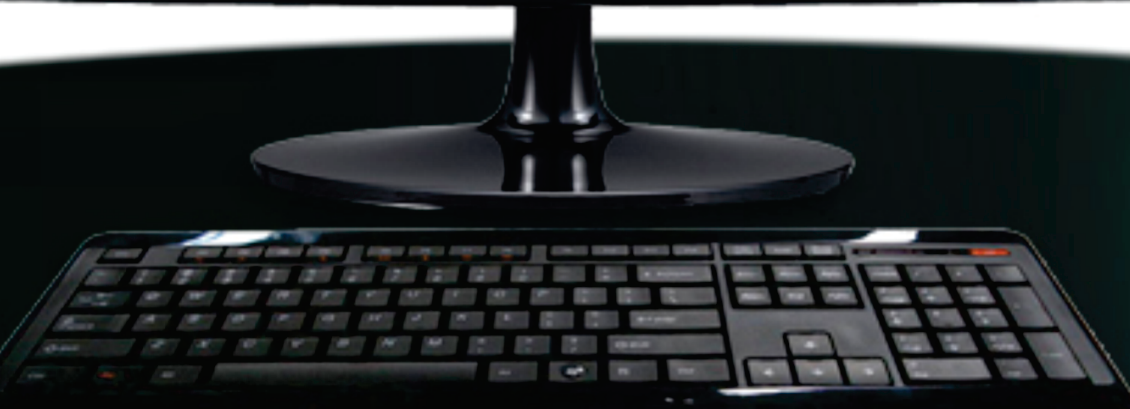

